\title{
Interactive Wallpaper
}

\author{
Azadeh Mohammadi \\ University of Toronto \\ Toronto, Ontario, Canada \\ azadeh.mohammadi@utoronto.ca
}

\author{
Taraneh Meshkani \\ Masters of Architecture, University of Toronto \\ Doctorate Design Candidate of Harvard University \\ taraneh.meshkani@utoronto.ca
}

\section{INTRODUCTION}

As an integral component of contemporary culture, technology creates a potential for the development of dynamic spaces. These spaces have an ability to interact and communicate with users.

To approach this idea, we proposed interactive wallpaper which responses to human activity. Wallpaper with a "transactive intelligence" (Ludovce, 2001) which can transform both the user and itself. The implication of such scheme can be wallpaper in spaces that lack sense of interaction and consequently create a dull environment. The best example of such space is a waiting room in a hospital.

\section{CONCEPTUAL IDEA}

The colour of the wallpaper in this project changes with the sound of the environment. The original colour of the wallpaper is blue, as the noise of the environment increases, the colour changes to warmer colour to the extent of red. With this effect, the wallpaper brings attention to the noise level of the room and encourages users to decrease the sound level of their conversations. The transformation in the colour shows the ability of Architecture to teach us how to react in different environments.

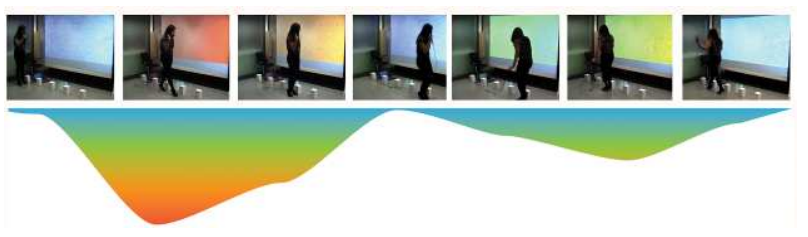

Aside from detecting the noise level of the room with the use of sound sensors the wallpaper also responses to the movement of users in the space. To develop the idea of interactive wallpaper, we chose design patterns of Persian tiles as a model of our design. The pattern of these tiles consists of complex geometries which come from the Islamic art. However, it is made from simple geometrical shape such as circles, squares, triangles and etc. The use of simple form to create complex patterns and the repetition of the geometric ornamentation has encouraged us to implement them in our project.

\section{PROTOTYPE}

For our prototype, we chose one example of geometric patterns and kept its guidelines to create our final design. Afterwards, we collected all the data from our pattern (points which are intersection of lines, squares, lozenges, starts and etc) and analyzed each of them based on changes in scale, movement and rotation. At the end we chose the points of our pattern to alter with the movement of users. The wallpaper consists of tiled layout. In each tile a distance sensor is embedded. The points in the pattern start to bounce when a user walks in front of a tile. This interaction distorts the pattern and changes its symmetry. The interactive pattern of the wallpaper creates an entertaining experience throughout the built environment.

Consequently, these interactive patterns offer "the possibility of a dynamic expression of the character of a given space or aggregation of spaces that is not predetermined by the designer, but emerges from occupation and use" (Moere, 2005).

\section{REFERENCES}

Ludovico, A. (2001) Marcus Novac interview. http://www.neural.it/english/marcosnovak.htm

Moere, A. (2005) The Symbiosis between Design \& Information Visualization. Key Centre of Design Computing \& Cognition School of Architecture. Design Science \& Planning, University of Sydney, Australia. 

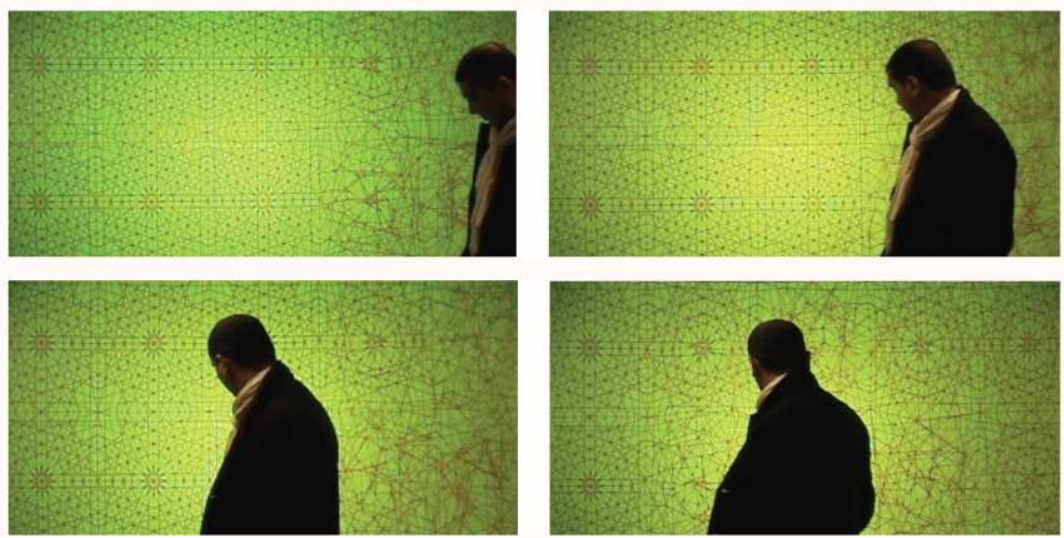